\title{
Adverse events of NOTES mediastinoscopy compared to conventional video-assisted mediastinoscopy: a randomized survival study in a porcine model
}

Authors

Institutions
Henry Córdova', Georgina Cubas², Marc Boada ${ }^{3}$, Cristina Rodríguez de Miguel' ${ }^{1}$, Graciela Martínez-Palli², Josep M. Gimferrer ${ }^{3}$, Gloria Fernández-Esparrach ${ }^{1}$

${ }^{1}$ Department of Gastroenterology, Hospital Clínic, University of Barcelona, Barcelona, Spain

${ }^{2}$ Anesthesiology Department, Hospital Clínic, University of Barcelona, Barcelona, Spain

3 Department of Thoracic Surgery, Hospital Clínic, University of Barcelona, Barcelona, Spain submitted 23. March 2015 accepted after revision 16. June 2015

\section{Bibliography}

Dol http://dx.doi.org/ 10.1055/s-0034-1392599

Published online: 11.8.2015

Endoscopy International Open 2015; 03: E571-E576

(c) Georg Thieme Verlag KG Stuttgart · New York

E-ISSN 2196-9736

Corresponding author

G. Fernández-Esparrach, MD PhD

Department of Gastroenterology ICMDiM

Hospital Clínic

Villarroel 170

08036 Barcelona

Spain

Fax: +34-93-2279387

mgfernan@clinic.ub.es
Background: Safety is a concern in natural orifice transluminal endoscopic surgery (NOTES) mediastinoscopy. The objective of this study was to compare the safety of NOTES mediastinoscopy with video-assisted mediastinoscopy (VAM).

Methods: Twenty-four pigs were randomly assigned to NOTES or VAM. Thirty-minute mediastinoscopies were performed with the identification of seven predetermined structures. The animals were euthanized after 7 days and necropsy was performed.

Results: Mediastinoscopy was not possible in one animal in each group. There were more intraoper-

\section{Introduction}

\section{$\nabla$}

Natural orifice transluminal endoscopic surgery (NOTES) has been demonstrated to be feasible in the mediastinum $[1,2]$. It has been suggested that the benefits of transesophageal access to the mediastinum or thoracic cavity might be even greater than intra-abdominal NOTES [3]. Conventional mediastinum surgery requires a relatively large incision to obtain access to the targeted organs. Conventional video-assisted mediastinoscopy (VAM) has overcome some of the inconveniences of conventional surgery because incisions are smaller, incision-related adverse events are reduced, and recovery is faster and less painful. However, VAM is associated with adverse events (for example, vessel injury and bleeding, and bronchial rupture) [4], and access to the posterior mediastinum and hilar regions is limited. Because thoracic NOTES allows access to the posterior, the middle mediastinum, and the pretracheal plane, it could, in theory, be preferable to VAM.

Initial experimental studies in NOTES mediastinoscopy showed a high rate of complications (pneumothorax 25\%, infections 20\%) [5,6] but there is no comparative study with VAM. Following preliminary experiments that demonstrated the feasibility of transesophageal NOTES [2], we ative adverse events with NOTES than VAM ( 7 vs. 2, $P=0.04$ ); hemorrhage was the most frequent adverse event (4 and 1, respectively). At necropsy, pathological findings were observed in 13 animals ( 9 NOTES and 4 VAM; $P=0.03$ ). Inflammatory parameters were not different between groups and were not related to adverse events. Conclusion: Systematic NOTES mediastinoscopy is possible and comparable to VAM in terms of number of organs identified and inflammatory impact. However, the safety profile of NOTES mediastinoscopy has to be improved before it can be adopted in a clinical setting.

performed this study to determine whether perioperative complications or postoperative infections would be insurmountable obstacles to pursuing this promising technique.

For this reason, the main aim of this study was to compare the safety of systematic transesophageal NOTES mediastinoscopy with conventional VAM. Other secondary objectives were to compare the efficacy of mediastinoscopy, inflammatory effects, and bacterial colonization.

\section{Materials and methods \\ $\nabla$ \\ Animals}

Experiments were performed in female Yorkshire pigs. The study was conducted at the University of Barcelona Medical School's animal facilities and was approved by the Animal Research Committee at the University of Barcelona. All animals were treated humanely. Animals underwent a 2-day quarantine and acclimation period. All procedures were performed under general anesthesia, using desflurane and endotracheal intubation.

\section{Study protocol}

Between February and November 2011, a total of 24 animals were randomized using random num- 
bers into NOTES mediastinoscopy $(n=12)$ or VAM $(n=12)$. Preoperative, anesthesia, and postoperative care protocols were identical for both groups. A venous catheter was placed at the internal jugular and all animals received antibiotic prophylaxis with a single dose of intravenous ceftriaxone $(1 \mathrm{~g})$. NOTES procedures were performed by two experienced endoscopists, whereas VAM was performed by two thoracic surgeons.

The surgical procedure consisted of a 30-minute mediastinoscopy, with the identification of seven predetermined structures (carina, right pulmonary artery, superior cava vein, right atrium, porcine bronchium, right vagus nerve, and brachiocephalic vein). All VAM equipment was sterile whereas NOTES equipment underwent a high level disinfection with $25 \%$ glutaraldehyde solution (Instrunet, Laboratorios Inibsa, Spain). Mediastinal access and incision closure varied depending on the group.

\section{NOTES}

A gastroscope (GIF H180, Olympus Medical Systems ${ }^{\circledR}$, Europe, Hamburg, Germany) was inserted through the pig's mouth and a submucosal tunnel was made as follows: the esophageal lumen was secured with an overtube. Just distal to the end of the overtube, about $25 \mathrm{~cm}$ from the mouth and using the trachea as anatomical reference, the site of interest in the esophagus was located and $10 \mathrm{~mL}$ of normal saline was injected to create a submucosal cushion. Following the injection, a superficial $10-\mathrm{mm}$ incision was created in the mucosal layer of the esophagus with a needleknife (KD-V451 M, Olympus Europe, Hamburg, Germany) using the cutting mode (power $30 \mathrm{~W}$ ) of an electrosurgical unit (PSD30, Olympus Europe, Hamburg, Germany). Then, the endoscope was introduced into the submucosal space and very gently pushed toward the distal esophagus. The endoscope acts like a blunted dissector, and the submucosa is easily separated from the muscularis propria creating a submucosal tunnel of $10 \mathrm{~cm}$. At the end of the tunnel, a full-thickness incision was created through the muscularis propria with the needle-knife and the endoscope was inserted into the posterior mediastinum. Once the scope was moved into the pretracheal space, blunt dissection with a Fogarty balloon (B-V233P-A/B, Olympus Europe, Hamburg, Germany) was used to advance the scope until the carina was identified. Pneumomediastinum was created and maintained with $\mathrm{CO}_{2}$ insufflation through the endoscope. After the mediastinoscopy, the submucosal space was closed by applying 1 to 3 clips at the mucosal entry site.

\section{VAM}

Video-assisted mediastinoscopy was carried out according to a standard technique [7]. The thorax was prepared in a sterile manner. A suprasternal incision was made followed by digital dissection of the pretracheal fascia and introduction of a sterile video-assisted mediastinoscope (model Telecam SL 202120 20, Karl Storz-Endoskope, Tuttlingen, Germany). A metal blunttipped suction-coagulation device and graspers were used alternately for the dissection of mediastinal structures. After the surgery, the video mediastinoscope was withdrawn and the defect closed with staples.

\section{Postoperative care and necropsy}

After rapid recovery from the anesthesia, the animals were transferred to individual cages and immediately had unlimited access to water. Food was allowed 8 hours after surgery. All animals were closely monitored for signs of mediastinitis and sepsis during the next 7 days. On the seventh day, the animals were eutha- nized and examined for evidence of pleural fluid and signs of infection, such as thoracic or mediastinal abscess.

Data collection, cytokine analysis, and bacterial analysis Blood samples for complete blood count, C-reactive protein (CRP) and TNF- $\alpha$ analysis were collected at baseline, at 1 and 8 hours after the procedure, and on postoperative days (POD) 1, 2, and 7. Aliquots of plasma were frozen and stored at $-80^{\circ} \mathrm{C}$ until analysis. Concentrations of TNF- $\alpha$ were measured using a quantitative sandwich enzyme-linked immunosorbent assay (ELISA) kit (R\&D Systems, Abingdon, UK). The technique sensitivity was $3.7 \mathrm{pg} / \mathrm{mL}$. Blood cultures were also collected at the end of surgery and on POD 2. All cultures were processed following a standardized technique: volumes of 5 to $8 \mathrm{~mL}$ of the samples were inoculated into aerobic and anaerobic vials in a Bactec System Fluorimetric 9000 (Becton-Dickinson Microbiology Systems, Franklin Lakes, New Jersey, USA). The incubation period was 5 days before being discarded as negative. The identification of microorganisms isolated from positive culture results was performed according to conventional methods.

\section{Definition of adverse events}

An intrasurgical adverse event was defined as an event that required an unplanned therapeutic maneuver regardless of whether or not it prevented completion of the procedure.

At necropsy, all abnormalities found were also considered adverse events. Mediastinitis findings consisted of severe adhesions, abscesses, and exudates.

\section{Statistical analysis}

The sample size was calculated to achieve $90 \%$ power to detect a difference in the incidence of adverse events (primary outcome) of $20 \%$ and an $\alpha$ value of 0.05 . The calculation resulted in a required sample size of 12 swine in each group (a total of 24 swine). Endpoint measures used to compare the safety of the two procedures were number of intraoperative adverse events, abnormal behavior in the follow-up period, and pathological findings at necropsy. Secondary outcomes were: mediastinoscopy efficacy, inflammatory impact, and type of organisms that colonized the mediastinum (assessed by number of mediastinal structures identified, TNF- $\alpha$ and PCR, and blood cultures, respectively).

Quantitative variables were analyzed using the Mann-Whitney test (for simple comparisons) and qualitative variables were analyzed with the $x^{2}$ test with Yates correction and Fisher's test. Multiple comparisons were performed with the Kruskal-Wallis test for continuous data and a Tukey HSD test for pairwise comparisons. Multiple comparisons were performed with Fisher's exact test for categorical data.

Cytokine values were first analyzed including values from all animals. However, to eliminate baseline variability in cytokines, data were then expressed as the difference between each value and baseline. Data are expressed as median and range. A $P$ value $<0.05$ was considered statistically significant. Statistical analysis was performed with SPSS ver. 17.0 (SPSS Inc., Chicago, Illinois, USA).

\section{Results}

$\nabla$

Twenty-four animals weighing $31.3 \pm 1.9 \mathrm{~kg}$ (25 to $37.3 \mathrm{~kg}$ ) were included in this study. The characteristics of the procedures are summarized in Table 1. The intraprocedural adverse event 
Table 1 Characteristics of the mediastinoscopies performed.

\begin{tabular}{|lccc|}
\hline & NOTES & VAM & P value \\
\hline Weight, mean \pm SD $($ range), kg & $31.9 \pm 1.8(30-35)$ & $30.6 \pm 1.8(28-35)$ & 0.34 \\
\hline Incision time, mean \pm SD (range), min & $10 \pm 2.5(7-15)$ & $10.9 \pm 3(7-16)$ & 0.69 \\
\hline Number of visualized structures, mean \pm SD (range) & $6.7 \pm 0.5(6-7)$ & $6.4 \pm 0.5(6-7)$ & 0.67 \\
\hline Time for closure of the incision, mean \pm SD (range), min & $3.3 \pm 1.8(1-8)$ & $5.0 \pm 1.5(3-8)$ & 0.18 \\
\hline Total time for surgery, mean \pm SD (range), min & $41.7 \pm 6.9(23-52)$ & $45.9 \pm 3.2(43-52)$ & 0.42 \\
\hline Number of intrasurgical adverse events, $n(\%)$ & $7(58.4 \%)^{1}$ & $2(16.7 \%)^{2}$ & 0.04 \\
\hline
\end{tabular}

NOTES, natural orifice transluminal endoscopic surgery; VAM, video-assisted mediastinoscopy; SD, standard deviation.

${ }^{1}$ Four hemorrhage, two pneumothorax, one burn in the trachea.

${ }^{2}$ One hemorrhage and one cardiopulmonary arrest during the induction of anesthesia.

\begin{tabular}{|c|c|c|c|}
\hline & $\begin{array}{l}\text { NOTES } \\
(n=11)\end{array}$ & $\begin{array}{l}\text { VAM } \\
(n=11)\end{array}$ & $P$ value \\
\hline Weight gain, median (range), kg & $-0.29(-1.3$ to 2.85$)$ & $0.08(-0.45$ to 2.1$)$ & 0.39 \\
\hline Necropsy findings, $n$ (\%) & $9(81.8)$ & $4(36.4)$ & 0.03 \\
\hline Closing defects in the esophagus, $\mathrm{n}$ & 4 & 0 & \\
\hline Mediastinitis $^{1}, \mathrm{n}$ & 2 & 0 & \\
\hline Adhesions, $\mathrm{n}$ & 1 & 2 & \\
\hline Abscess in the esophagus, $\mathrm{n}$ & 1 & 0 & \\
\hline Pericardial effusion, $\mathrm{n}$ & 1 & 0 & \\
\hline Hematoma in the mediastinum, $n$ & 0 & 1 & \\
\hline Abscess in the surgical incision, $n$ & 0 & 1 & \\
\hline
\end{tabular}

NOTES, natural orifice transluminal endoscopic surgery; VAM, video-assisted mediastinoscopy.

${ }^{1}$ Defined by the presence of severe adhesions, abscesses, or exudates.

rate was higher in the NOTES group with seven events (58.4\%) (four hemorrhage, two pneumothorax, and one burn in the trachea) versus two $(16.7 \%)$ with VAM (one hemorrhage and one cardiopulmonary arrest during the induction of anesthesia) $(P=$ $0.04)$. One major hemorrhage in the NOTES group was noted immediately after the incision through the muscularis propria with the needle-knife, which could not be stopped endoscopically and required euthanasia of the animal. The other three episodes of hemorrhage were minor and were treated with conventional maneuvers (coagulation forceps). Pleural injury was visually confirmed in two animals and tension pneumothorax was relieved by insertion of a percutaneous catheter. The burn in the trachea was limited and did not require any intervention. The cardiopulmonary arrest in the VAM group was fatal and prevented starting the mediastinoscopy.

The 22 animals in which the surgery was completed survived until they were euthanized on day 7 . Weight loss was observed in 18 animals with no differences between the two groups. Two animals in the NOTES group developed clinical signs of infection (one with diarrhea and one with severe prostration).

The number of abnormal necropsy findings was higher in the NOTES group $(n=9,81.8 \%)$ than in the VAM group $(n=4,36.4 \%)$ $(P=0.03)$ ( Table 2). Necropsy confirmed mediastinitis in two animals in the NOTES group that had shown signs of infection during follow-up ( $\mathbf{O}$ Fig. $\mathbf{1}$ ). In four animals in the NOTES group, we observed defects in the muscular layer of the esophagus without any macroscopic sign of mediastinitis.

The overall number of adverse events (intraoperative and at the end of follow-up) was higher in the NOTES group than in the VAM group ( 16 vs $6, P=0.002$ ).

Regarding the efficacy of mediastinoscopy, precarinal and paratracheal regions were accessed without difficulty in both groups and at least six of seven structures were seen in all cases.
TNF- $\alpha$ levels, white blood cells, hemoglobin, and hematocrit were similar among both groups at baseline and during the follow-up period ( $\bullet$ Fig. 2). Serum TNF- $\alpha$ values increased in both groups 1 hour after the procedure and were normal again at 48 hours. Similarly, 8 hours after the procedure, white blood cell values and CRP demonstrated an increase in both groups returning to baseline at POD 7.

Positive culture results were seen in both groups at the end of the procedure and in all animals on POD $2(\bullet$ Table 3$)$. The most common organisms that colonized the mediastinum were Gram-positive cocci. Most of the bacteria isolated in our study are part of the normal oral and skin flora in swine (http://www.bacterio. cict.fr/bacdico/).

\section{Discussion}

$\nabla$

Recently, systematic transesophageal mediastinoscopy has been described [2] as an excellent technique for the visualization of mediastinal structures located in the middle mediastinum and pretracheal plane, which is comparable to images obtained during VAM. However, this is the first study comparing the performance of NOTES mediastinoscopy with conventional VAM. Our study protocol included only a timed mediastinoscopy and we did not perform any complex surgery or try to target any difficult structures. In this study, the number of structures identified was similar in both groups.

Inadvertent high pressure pneumomediastinum and pneumothorax have been major adverse events reported since the introduction of thoracic NOTES $[2,5,6,8,9]$. This event can be explained by the characteristics of the endoscopic access technique, which includes the dissection of the connective tissue located in the pretracheal space. During this maneuver, the parietal pleura 


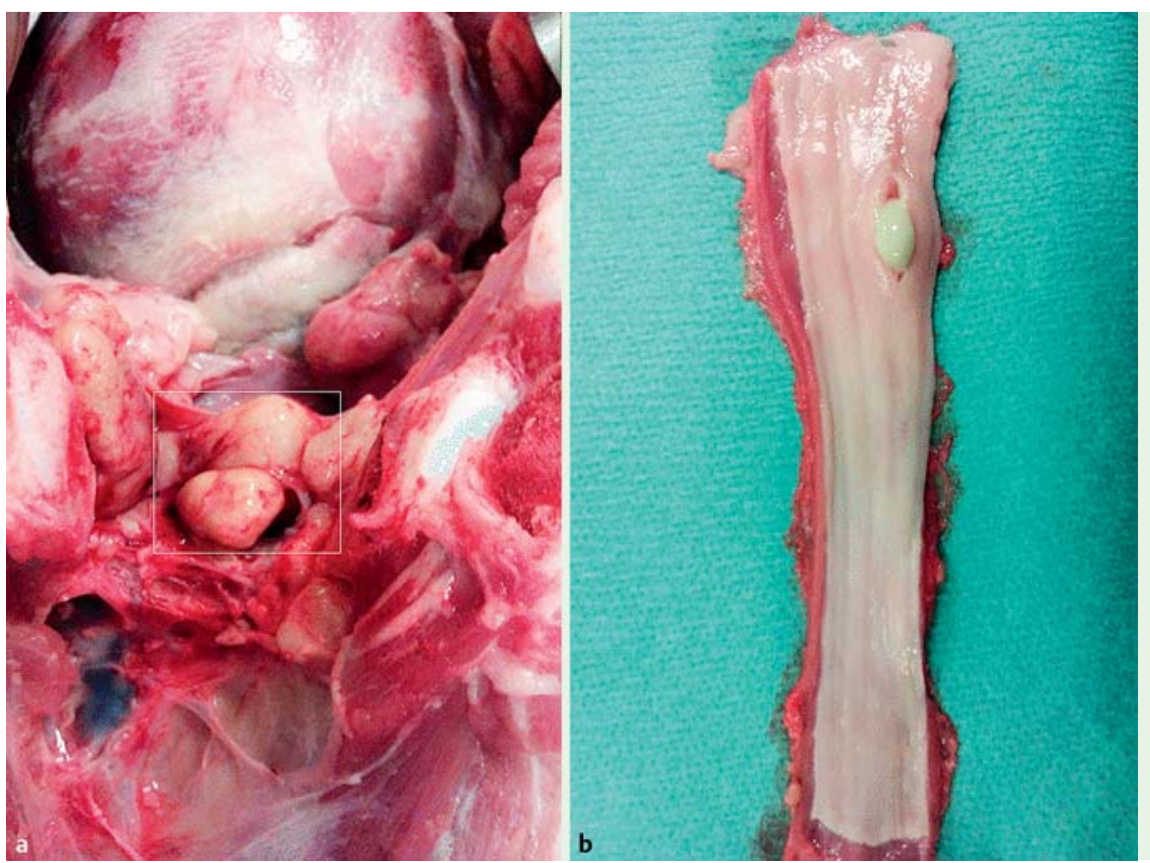

Fig. 1 Some necropsy findings. a Multiple abscesses can be seen (open white rectangle) in the mediastinum. $\mathbf{b}$ Gross esophageal specimen with an intramural abscess within the mucosa at the esophageal incision.

can be injured and the insufflation of $\mathrm{CO}_{2}$ can lead to a tension pneumothorax that persists even after aspiration through the endoscope. This is probably due to a valve effect and requires immediate drainage. However, close monitoring of both hemodynamic and respiratory parameters allows early detection and treatment of pneumothorax and prevents the subsequent fatal outcome [9].

We chose the method of submucosal tunneling to reach the mediastinum through the esophagus because this has demonstrated its efficacy as a method of closure as well [2]. However, macroscopic examination at necropsy revealed some defects in the muscular layer that, otherwise, did not have any negative clinical impact. It could be argued that 1 week was not sufficient time to achieve complete healing and these findings could be judged as normal in the healing process, explaining why we did not find signs of mediastinitis in these animals. In fact, Fritscher-Ravens et al. [3] performed an endoscopic inspection of esophageal mucosal incision after 2 weeks and before euthanasia, finding some remaining superficial ulceration in the area of incision of the esophagus. Based on this observation, these authors decided to keep the animals alive for $4-6$ weeks. At this time, endoscopy revealed a well healed endoluminal esophagus in all animals without any ulcerations or obvious visible marks, and autopsy revealed neither macroscopic nor microscopic mediastinitis nor pericarditis. However, another study assessing the early healing of gastric and colonic incisions showed complete macroscopic healing 1 week after NOTES procedures suggesting a different timing for each organ [10].

Even with submucosal tunneling, the transesophageal approach to the thoracic cavity is risky due to the possible trauma to surrounding structures [11]. In the present study, the incidence of intrasurgical adverse events was significantly higher in transesophageal access, with bleeding as the most frequent event. All apart from one bleeding could be stopped with standard endoscopic maneuvers but we were very strict with the definition of adverse events to avoid an underestimation. As described in the initial report on submucosal endoscopy with a mucosal flap valve [11], we used a needle-knife to cut the muscular layer. However, the use of more precise instruments such as a triangle-tip knife or different electrosurgical settings could avoid these episodes of minor bleeding. Another solution is to assist the transesophageal access with EUS or a CT-based image registration system [12,13], or even with a single transthoracic trocar [14]. In a comparative study of NOTES alone versus EUS-assisted NOTES procedures, Fritscher-Ravens et al. [12] found that assisted procedures were superior in gaining access, identifying structures, and avoiding major adverse events. In a recent prospective, randomized, controlled trial of blind mediastinoscopy against CT-based image registered procedures, our group has demonstrated that guidance could be useful in selected scenarios; however, the incidence of adverse events was high in both groups [13].

Several infections that led to severe mediastinitis and death in some cases [6] have been reported previously. Additionally, there are some controversies about the need for antibiotics, the duration of fasting, and endoscope sterilization. Antibiotic therapy has been reported in the majority of studies, and antibiotics have been administered not only pre-interventional (as in the present study) but also peri- and postoperatively [15]. However, because blood cultures are not performed routinely, it is very difficult to assess the efficacy of different strategies. Although clinical signs of infection were only seen in two animals, blood cultures were positive in all subjects and abnormal findings were significantly higher at necropsy in the NOTES group. The fact that most of the bacteria isolated in our study are part of the normal skin flora of swine could explain the difficulties in maintaining sterile access for subsequent blood samples. Therefore, we recommend caution when transferring the results from animal experiments to humans in whom a perforation of the esophagus causes severe adverse events or even death in at least $30-50 \%$ of cases [15]. However, recent experience with peroral endoscopic myotomy (POEM), which uses the same submucosal tunneling to access the muscular layer, has shown a good safety profile [16].

Similar to previous studies in abdominal NOTES [17], all proinflammatory mediators measured were increased within 8 hours after NOTES mediastinoscopy. However, these levels were not dif- 

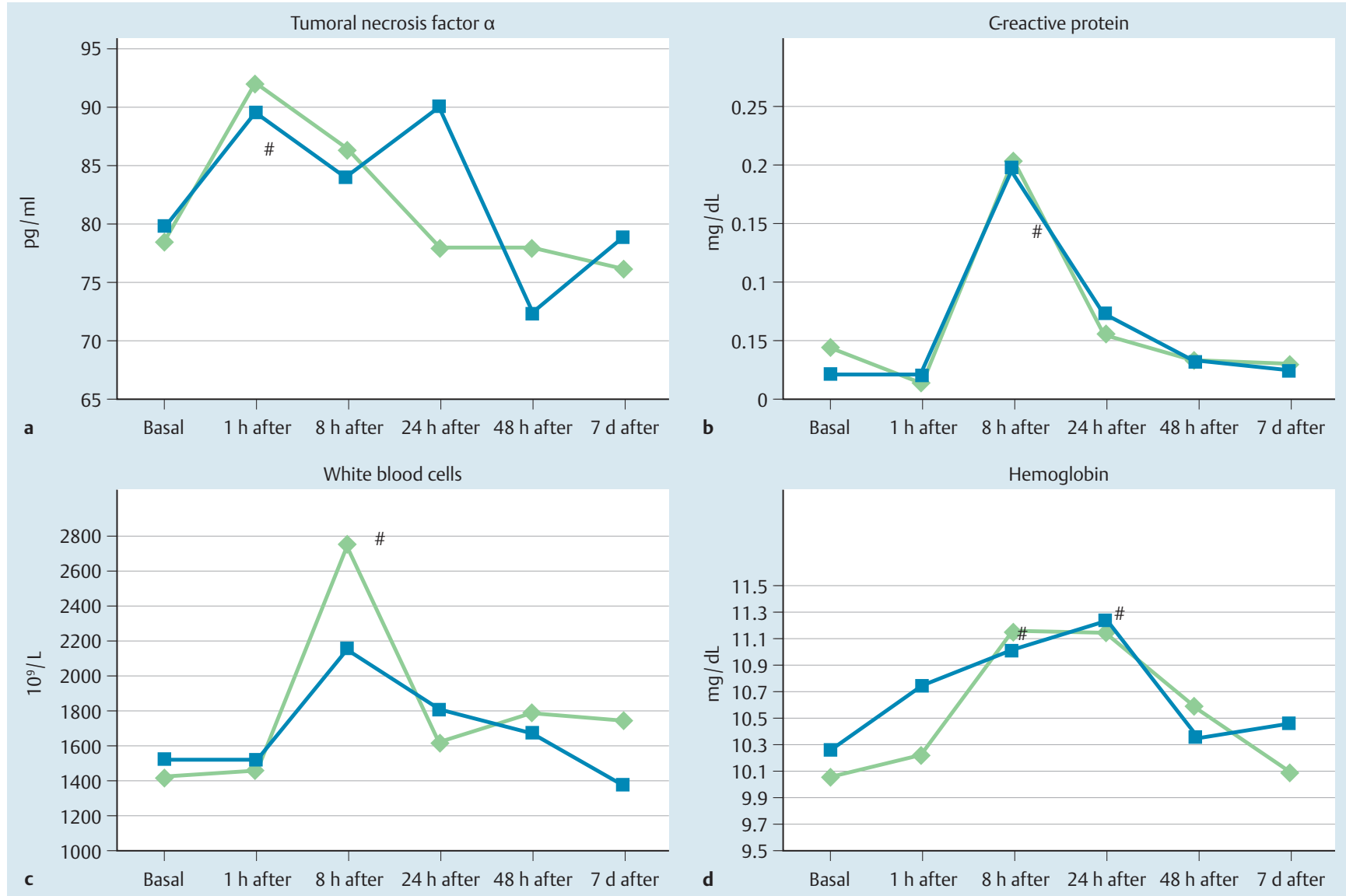

Fig. 2 Inflammatory changes. Group comparison of TNF $\alpha, C$ reactive protein, white blood cells, and hemoglobin values over time ( $\#, P<0.05$ from baseline).

\begin{tabular}{|llll|}
\hline & NOTES (n=12) & $\begin{array}{l}\text { VAM } \\
(\mathbf{n = 1 2 )}\end{array}$ & P value \\
\hline Basal positive cultures & & $4 / 11$ & 0.56 \\
\hline Positive cultures on POD 2 & $3 / 12$ & $9 / 9$ & 0.37 \\
\hline Two or more species isolated on POD 2 & $10 / 11$ & 4 & 0.96 \\
\hline Abscesses & 5 & 1 & NP \\
\hline Mediastinitis & 1 & 0 & 0.13 \\
\hline
\end{tabular}

Table 3 Bacterial infection after NOTES mediastinoscopy and VAM.

NOTES, natural orifice transluminal endoscopic surgery; VAM, video-assisted mediastinoscopy; NP, not possible to calculate a Chi squared value because the variable is a constant; POD, postoperative day.

ferent compared with VAM and were not related to adverse events. Similarly, animals in both groups lost weight during the follow-up period, which could reflect the inflammatory impact of this type of surgery and could have been higher in the case of a non-minimally invasive surgery.

Gastrointestinal endoscopists are familiar with the mediastinum. Incisionless access to the mediastinum has been used for years for mediastinal lymph node sampling using endoscopic ultrasonography (EUS), and has demonstrated a solid safety profile and accuracy [18]. EUS-guided fine needle aspiration is able to routinely access lymph node stations located in the posterior and middle mediastinum, but is unable to reach peribronchial and hilar stations and the pretracheal plane. On the other hand, VAM can explore these ganglionar stations but cannot reach the posterior mediastinum. Although we only explored the pretracheal space and the middle mediastinum in this study because we were comparing NOTES mediastinoscopy with VAM, the endoscope accessed the posterior mediastinum through the esophageal pos- terior wall, demonstrating that transesophageal mediastinoscopy could be an alternative approach in achieving a complete exploration of the mediastinum. The feasibility of transesophageal NOTES opens new possibilities of less invasive access to the mediastinum and thoracic cavity, and has influenced the evolution of therapeutic endoscopy, and POEM is a perfect example of this $[16,19]$. It is mandatory that physicians who perform esophageal endoscopic procedures should know the range of potential mediastinal adverse events and their management.

This study has several limitations. First, the anatomy of pigs' mediastinum is not the same as in humans, in which the right atrium, vagus nerve, and brachiocephalic vein cannot be visualized by VAM. Second, the number of animals included was relatively small but it was the result of a power calculation and the two animals that were sacrificed before the completion of mediastinoscopy were not excluded and were counted as adverse events. Third, some findings at necropsy are unique for each surgical technique. Fourth, we did not use a standardized nomenclature 
for adverse events. And fifth, 1-week survival could be too short to evaluate the complete healing of esophageal incisions.

In conclusion, systematic NOTES mediastinoscopy is possible and comparable to VAM in terms of number of organs identified and inflammatory impact. However, the safety profile of NOTES mediastinoscopy has to be improved before it can be adopted in a clinical setting.

\section{Competing interests: None}

\section{Acknowledgments}

This research project was supported by a grant from the Ministerio de Ciencia e Innovación (SAF2010-15635). Dr Córdova was supported by the Instituto Nacional de Salud Carlos III.

\section{References}

1 Turner BG, Gee DW, Cizginer S et al. Endoscopic transesophageal mediastinal lymph node dissection and en bloc resection by using mediastinal and thoracic approaches (with video). Gastrointest Endosc 2010; 72: $831-835$

2 Córdova H, Martinez-Pallí G, Fernández-Esparrach G. Feasibility of systematic transesophageal endoscopic mediastinoscopy. Thorac Cardiovasc Surg 2013; 61: 486-488

3 Fritscher-Ravens A, Patel K, Ghanbari A et al. Natural orifice transluminal endoscopic surgery (NOTES) in the mediastinum: long-term survival animal experiments in transesophageal access, including minor surgical procedures. Endoscopy 2007; 39: 870-875

4 Yan TD, Black D, Bannon PG et al. Systematic review and meta-analysis of randomized and nonrandomized trials on safety and efficacy of video-assisted thoracic surgery lobectomy for early-stage non-small cell lung cancer. J Clin Oncol 2009; 16: 2152 - 2165

5 Willingham FF, Gee DW, Lauwers GY et al. Natural orifice transesophageal mediastinoscopy and thoracoscopy. Surg Endosc 2008; 22: 1042 1047

6 Gee DW, Willingham FF, Lauwers GY et al. Natural orifice transesophageal mediastinoscopy and thoracoscopy: a survival series in swine. Surg Endosc 2008; 22: 2117-2122
7 Venissac N, Alifano M, Mouroux J. Video-assisted mediastinoscopy: experience from 240 consecutive cases. Ann Thorac Surg 2003; 76: 208 212

8 von Delius S, Wilhelm D, Feussner $H$ et al. Natural orifice transluminal endoscopic surgery: cardiopulmonary safety of transesophageal mediastinoscopy. Endoscopy 2010; 42: 405-412

9 Navarro-Ripoll R, Córdova H, Rodríguez-D’Jesús A et al. Cardiorespiratory impact of transesophageal endoscopic mediastinoscopy compared to cervical mediastinoscopy: a randomized experimental study. Surg Innov 2014; 21: 487-495

10 Mathews JC, Chin MS, Fernandez-Esparrach G et al. Early healing of transcolonic and transgastric natural orifice transluminal endoscopic surgery access sites. J Am Coll Surg 2010; 210: 480-490

11 Sumiyama K, Gostout CJ, Rajan E et al. Transesophageal mediastinoscopy by submucosal endoscopy with mucosal flap safety valve technique. Gastrointest Endosc 2007; 65: 679-683

12 Fritscher-Ravens A, Ghanbari A, Cuming $T$ et al. Comparative study of NOTES alone vs. EUS-guided NOTES procedures. Endoscopy 2008; 40: 925-930

13 Córdova H, San José Estépar R, Rodríguez-D'Jesús A et al. Comparative study of NOTES alone versus NOTES guided by a new image registration system for navigation in the mediastinum: a study in a porcine model. Gastrointest Endosc 2013; 77: 102 - 107

14 Moreira-Pinto J, Ferreira A, Miranda A et al. Transesophageal pulmonary lobectomy with single transthoracic port assistance: study with survival assessment in a porcine model. Endoscopy 2012; 44: $354-$ 361

15 Grund KE, Lehmann TG. Transesophageal NOTES - a critical analysis of relevant problems. Minim Invasive Ther Allied Technol 2010; 19: $252-256$

16 Inoue H, Minami H, Kobayashi $Y$ et al. Peroral endoscopic myotomy (POEM) for esophageal achalasia. Endoscopy 2010; 42: 265-271

17 Guarner-Argente C, Beltrán M, Martínez-Pallí $G$ et al. Infection during natural orifice transluminal endoscopic surgery peritoneoscopy: a randomized comparative study in a survival porcine model. J Minim Invasive Gynecol 2011; 18: 741 - 746

18 Silvestri GA, Hoffmann BJ, Bhutani MS et al. Endoscopic ultrasound with fine needle aspiration in the diagnosis and staging of lung cancer. Ann Thorac Surg 1996; 61: 1441 -1446

19 Pasricha PJ, Hawari R, Ahmed I et al. Submucosal endoscopic esophageal myotomy: a novel experimental approach for the treatment of achalasia. Endoscopy 2007; 39: 761 - 764 